\title{
Synthesis, Characterization and Biological Activity of 2-Methyl-3- aminoquinazolin-4(3H)-ones Schiff Bases
}

\author{
S. K. Sahu, ${ }^{* a}$, Md. Afzal Azam, ${ }^{b}$ M. Banerjee,${ }^{a}$ S. Acharrya, ${ }^{d}$ C. C. Behera ${ }^{a}$ and S. Si ${ }^{c}$ \\ ${ }^{a}$ University Department of Pharmaceutical Sciences, Utkal University, Vani Vihar, Bhubaneswar-751004, Orissa, India \\ ${ }^{b}$ Department of Pharmaceutical Chemistry, J. S. S., College of Pharmacy, Ootacamund-643001,Tamil Nadu, India
}

'School of Pharmaceutical Sciences, ITER, Bhubaneswar, Orissa, India

${ }^{d}$ Institute of Pharmacy and Technology, Salipur, Orissa, India

\begin{abstract}
$\mathrm{O}$ tratamento de 3-amino-2-metilquinazolina/6-bromo-2-metilquinazolina-4(3H)-onas, 2a,b, com 2,3-indolinediona na presença de traços de ácido acético glacial forneceu $3-\left\{\left(2^{\prime}\right.\right.$-oxo- $1^{\prime}, 2^{\prime}$-dihidroindol-3'-ilideno)amino\}-2-metilquinazolina/6-bromo-2-metilquinazolina-4-( $3 H$-onas, 3a,b, as quais foram condensadas com várias aminas secundárias e formaldeído em etanol para originar compostos do tipo 3-\{(1'-alquil/arilaminometil-2'-oxo-1', $2^{\prime}$-di-hidroindol-3'-ilideno $)$ amino $\}-2-$ metil-6-quinazolin-4-(3H)-onas, $\mathbf{4} \mathbf{a}_{\mathbf{1 - 6}}$ e $\mathbf{4} \mathbf{b}_{\mathbf{1 - 6}}$. Os compostos sintetizados foram caracterizados por análise elementar, espectroscopia no infravermelho, RMN ${ }^{1} \mathrm{H}$ e espectrometria de massas. Adicionalmente, foram investigadas suas atividades antimicrobiana, analgésica, anti-inflamatória e anti-helmíntica. Os resultados das atividades biológicas revelaram que os compostos $\mathbf{4 a}_{3}, \mathbf{4} \mathbf{a}_{4}$ e $\mathbf{4} \mathbf{b}_{\mathbf{6}}$ exibiram atividades analgésica e anti-inflamatória significativas. Os compostos $\mathbf{4} \mathbf{b}_{5}$ e $\mathbf{4 b}_{\mathbf{6}}$ apresentaram atividade anti-helmíntica, quando testados frente a Pheretima posthuma.
\end{abstract}

The 3-amino-2-methylquinazoline/6-bromo-2-methylquinazoline-4(3H)-ones, 2a,b, on treatment with 2,3-indolinedione in the presence of traces of glacial acetic acid yielded 3-\{(2'-oxo$1^{\prime}, 2^{\prime}$-dihydroindole-3'-ylidene)amino $\}$-2-methylquinazolin/6-bromo-2-methylquinazolin-4-( $3 H$ ones, $\mathbf{3 a}, \mathbf{b}$, which on condensation with various secondary amines and formaldehyde in ethanol afforded title compounds $3-\left\{\left(1^{\prime}\right.\right.$-alkyl/arylaminomethyl-2'-oxo- $1^{\prime}, 2^{\prime}$-dihydroindole- $3^{\prime}$-ylidene $)$ amino -2-methyl-6-quinazolin-4-(3H)-ones, $\mathbf{4 a}_{\mathbf{1 - 6}}$ and $\mathbf{4} \mathbf{b}_{\mathbf{1 - 6}} . \mathbf{C}, \mathrm{H}, \mathrm{N}$ analysis, infrared spectroscopy, ${ }^{1} \mathrm{H}$ NMR, and mass spectroscopy allowed the identification of the synthesized compounds, which were investigated for their antimicrobial, analgesic, anti-inflammatory and antihelmintic activities. The results of the biological activities revealed that the compounds $\mathbf{4} \mathbf{a}_{3}, \mathbf{4} \mathbf{a}_{\mathbf{4}}$ and $\mathbf{4} \mathbf{b}_{6}$ exhibited significant analgesic and anti-inflammatory activities. Compounds $\mathbf{4} \mathbf{b}_{5}$ and $\mathbf{4} \mathbf{b}_{\mathbf{6}}$ showed antihelmintic activity when tested against Pheretima posthuma.

Keywords: 2-methylquinazolin-4(3H)-one, bromoderivative, 2,3-indolinedione, Mannich base, antimicrobial

\section{Introduction}

In recent years there has been an increased interest in the chemistry of quinazoline- $4(3 H)$-ones because of their biological significance. Different types of quinazoline$4(3 \mathrm{H})$-ones exhibit a wide spectrum of biological activities including antihelmintic, antimicrobial and anti-inflammatory activity ${ }^{1-4}$ Certain Schiff and Mannich bases of 2,3-indolinedione derivatives have been reported to possess significant antimicrobial and anti-inflammatory

*e-mail: tutu_kh@yahoo.com activity. ${ }^{5-9}$ In view of these findings it appeared of interest to synthesize a series of novel Schiff bases of 2-methylquinazoline/6-bromo-2-methylquinazoline4(3H)-ones containing 2,3-indolinedione or Mannich bases of 2,3-indolinedione residues with the aim of obtaining some novel heterocyclic systems with potentially enhanced antimicrobial, analgesic, anti-inflammatory and antihelmintic activities. In the present investigation, a new series of novel $3-\left\{\left(1^{\prime}\right.\right.$-substituted aminomethyl2'-oxo-1',2'-dihydro-indole-3'-yl-idene)amino \}-2methylquinazolin/6-bromo-2-methylquinazolin-4$(3 H)$-ones $\mathbf{4 a}_{\mathbf{1 - 6}}$ and $\mathbf{4} \mathbf{b}_{1-6}$ was prepared as per Scheme 1 


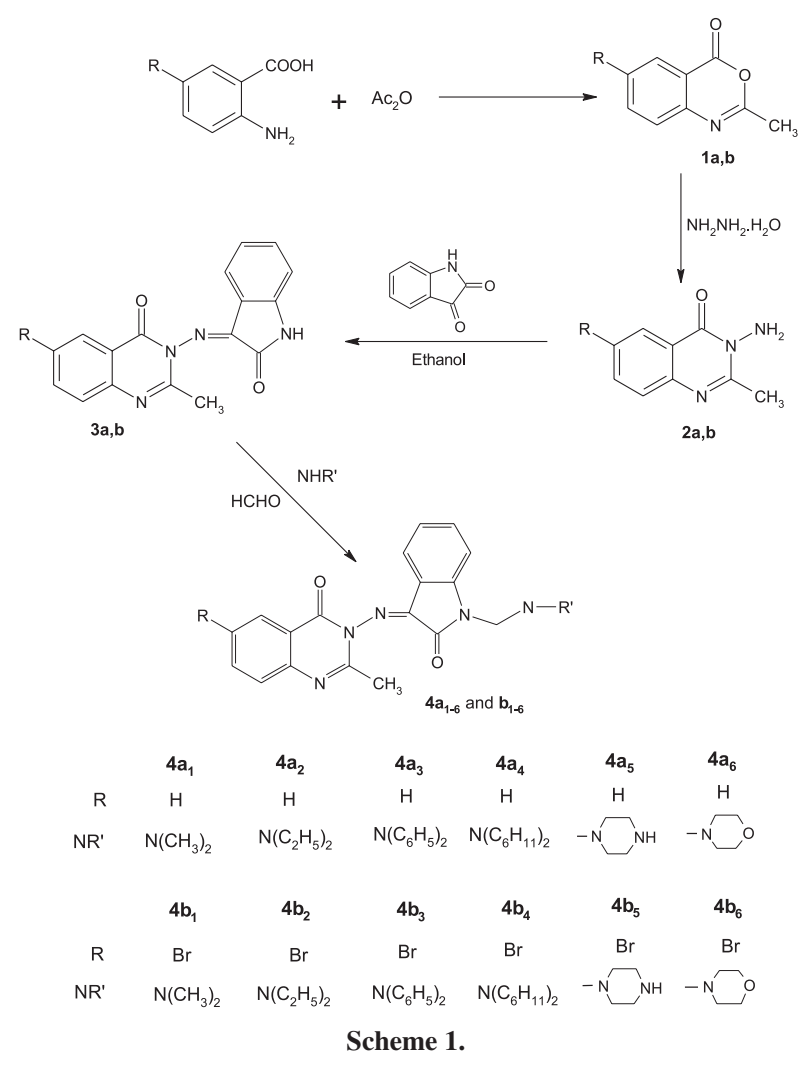

and screened for their antimicrobial, analgesic, antiinflammatory and antihelmintic activities.

\section{Results and Discussion}

\section{Chemical synthesis}

The starting material 3-amino-2-methylquinazolin-6bromo-2-methylquinazolin-4(3H)-ones $\mathbf{2 a , b}$ were prepared according to literature methods, ${ }^{10,11}$ from the corresponding anthranilic acid. 3-Aminoquinazolinone derivatives 2a,b were used as precursors for further reaction with the purpose of synthesizing several heterocyclic compounds attached to the 3-methylquinazolin-4(3H)-one moiety. Thus, condensation of $\mathbf{2 a , b}$ with 2,3-indolinedione in the presence of trace amount of glacial acetic acid, furnished the corresponding Schiff bases 3a,b. The Schiff bases thus obtained were treated in ethanol with formalin and various secondary amines to afford the corresponding Mannich bases $\mathbf{4 a}_{\mathbf{1 - 6}}$ and $\mathbf{4} \mathbf{b}_{\mathbf{1 - 6}}$. Analytical data, FT-IR, ${ }^{1} \mathrm{H}$ NMR and mass spectra confirmed the structures of the new compounds $\mathbf{4} \mathbf{a}_{1-6}$ and $\mathbf{4} \mathbf{b}_{1-6}$. Compounds $\mathbf{3 a}, \mathbf{b}$ showed absorption bands at $3472 \mathrm{~cm}^{-1}$ for NH group and at 1727 and $1670 \mathrm{~cm}^{-1}$ due to $\mathrm{C}=\mathrm{O}$ stretching vibrations. Also, the ${ }^{1} \mathrm{H}$ NMR spectra of compounds $\mathbf{3} \mathbf{a}$ and $\mathbf{3 b}$ showed a singlet at $\delta 10.63$ and $10.53 \mathrm{ppm}$, respectively for the NH hydrogens, confirming the formation of Schiff bases 3a, $\mathbf{b}$.
The mass spectra of 3a showed molecular ion peak $\mathrm{M}^{+}$at $\mathrm{m} / \mathrm{z} 304$ corresponding to the molecular formula $\mathrm{C}_{17} \mathrm{H}_{12} \mathrm{~N}_{4} \mathrm{O}_{2}$. The infrared spectra of compounds $\mathbf{4} \mathbf{a}_{\mathbf{1}-6}$ and $\mathbf{4} \mathbf{b}_{1-6}$ were characterized by the disappearance of the NH absorption band. Also, the ${ }^{1} \mathrm{H}$ NMR spectra of title compounds $\mathbf{4} \mathbf{a}_{1-6}$ and $\mathbf{4} \mathbf{b}_{1-6}$ showed the presence of methylene group and the aromatic hydrogens all appeared at the expected chemical shifts (see Experimental). The mass spectra of $\mathbf{4} \mathbf{a}_{1}$ showed the molecular ion peak $\mathbf{M}^{+}$at $m / z 361$ corresponding to the molecular formula $\mathrm{C}_{20} \mathrm{H}_{19} \mathrm{~N}_{5} \mathrm{O}_{2}$.

\section{Biological study}

\section{Antimicrobial activity}

Compounds $\mathbf{4} \mathbf{a}_{1-6}$ and $\mathbf{4} \mathbf{b}_{1-6}$ were assayed for their antibacterial activity against Staphylococcus aureus, Staphylococcus faecalis, Escherichia coli, and Salmonella typhi and for antifungal activities against Candida albicans and Aspergillus niger by cup plate method. ${ }^{12}$ Further, their minimum inhibitory concentration values against these microorganisms were determined by serial dilution method. ${ }^{13}$ The test microorganisms were obtained from Department of Microbiology, OUAT, Orissa, India. Muller Hinton agar plates $\left(37^{\circ} \mathrm{C}, 24 \mathrm{~h}\right)$ and Sabouraud's dextrose agar plates $\left(26^{\circ} \mathrm{C}, 48-72 \mathrm{~h}\right)$ were used for the cultivation of bacteria and fungi, respectively. The results of antibacterial and antifungal activity tests are summarized in Tables 1 and 2 with standard drugs ciprofloxacin and clotrimazole for comparison. Most of the synthesized compounds were found to possess varied degrees of antibacterial activities as evident from their minimal inhibitory concentration (MIC). Compounds $\mathbf{4} \mathbf{b}_{5}$ and $\mathbf{4} \mathbf{b}_{6}$ were found to possess moderate activity against all the tested bacteria while all the tested compounds except $\mathbf{4} \mathbf{b}_{5}$ and $\mathbf{4} \mathbf{b}_{6}$ showed lower activity against Escherichia coli. Most of the synthesized compounds were found to possess moderate activity against tested fungi. However, no correlation was observed between different substituents at $1^{\prime}$ position of 2,3-indolinedione ring and antifungal activity. It is noted that bromination at 6 position of 2-methylquinazolin$4(3 H)$-one ring does not have any significant effect on antimicrobial activity.

\section{Analgesic and anti-inflammatory activity}

The synthesized compounds $\mathbf{4 a}_{\mathbf{1 - 6}}$ and $\mathbf{4} \mathbf{b}_{\mathbf{1 - 6}}$ were evaluated for analgesic and anti-inflammatory activities. Students $t$-test were performed to ascertain the significance of the exhibited activities. The test compounds $\left(100 \mathrm{mg} \mathrm{kg}^{-1}\right)$ and the standard drug indomethacin $\left(10 \mathrm{mg} \mathrm{kg}^{-1}\right)$ were administered in the 
Table 1. In vitro antimicrobial activities of compounds $\mathbf{4} \mathbf{a}_{\mathbf{1 - 6}}$ and $\mathbf{4} \mathbf{b}_{\mathbf{1 - 6}}$ against various bacteria and fungi

\begin{tabular}{|c|c|c|c|c|c|c|}
\hline \multirow{2}{*}{ Compound No. } & \multicolumn{6}{|c|}{ Zone of Inhibition $^{\mathrm{a}}(\mathrm{mm}) 100 \mu \mathrm{g} \mathrm{mL}^{-1}$} \\
\hline & S. $a$ & S.f & E. $c$ & S. $t$ & C. $a$ & A. $n$ \\
\hline $4 a_{1}$ & 16 & 18 & 20 & 19 & 14 & 17 \\
\hline $4 a_{2}$ & 13 & 15 & 16 & 15 & 13 & 19 \\
\hline $4 a_{3}$ & 18 & 18 & 20 & 16 & 17 & 18 \\
\hline $4 a_{4}$ & 19 & 18 & 19 & 17 & 18 & 21 \\
\hline $4 a_{5}$ & 20 & 21 & 22 & 20 & 19 & 20 \\
\hline $4 a_{6}$ & 20 & 22 & 26 & 23 & 21 & 24 \\
\hline $4 b_{1}$ & 13 & 16 & 16 & 16 & 19 & 18 \\
\hline $4 b_{2}$ & 11 & 17 & 18 & 19 & 19 & 19 \\
\hline $4 b_{3}$ & 13 & 17 & 17 & 17 & 21 & 17 \\
\hline $4 b_{4}$ & 14 & 16 & 19 & 17 & 19 & 21 \\
\hline $4 b_{5}$ & 22 & 23 & 21 & 25 & 24 & 24 \\
\hline $4 b_{6}$ & 21 & 23 & 27 & 23 & 24 & 22 \\
\hline Ciprofloxacin & 29 & 31 & 32 & 26 & - & - \\
\hline Clotrimazole & - & - & - & - & 28 & 27 \\
\hline DMF & - & - & - & - & - & - \\
\hline
\end{tabular}

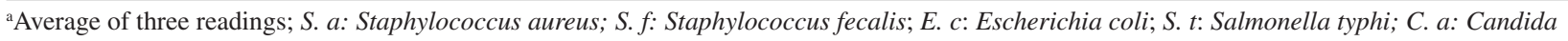
albicans; A. n: Aspergillus niger.

Table 2. Minimum inhibitory concentrations of compounds $\mathbf{4} \mathbf{a}_{1-6}$ and $\mathbf{4} \mathbf{b}_{\mathbf{1 - 6}}$ against various bacteria and fungi

\begin{tabular}{|c|c|c|c|c|c|c|}
\hline \multirow{2}{*}{ Compound No. } & \multicolumn{6}{|c|}{ MIC values ${ }^{\mathrm{a}} \mu \mathrm{g} \mathrm{mL}^{-1}$} \\
\hline & S. $a$ & S. $f$ & E. $c$ & S. $t$ & C. $a$ & A. $n$ \\
\hline $4 a_{1}$ & 25 & 25 & 50 & 25 & 25 & 25 \\
\hline $4 a_{2}$ & 25 & 25 & 50 & 25 & 25 & 25 \\
\hline $4 a_{3}$ & 25 & 50 & 50 & 25 & 50 & 25 \\
\hline $4 a_{4}$ & 25 & 50 & 25 & 50 & 25 & 25 \\
\hline $4 a_{5}$ & 50 & 25 & 50 & 25 & 25 & 25 \\
\hline $4 a_{6}$ & 25 & 25 & 50 & 50 & 25 & 25 \\
\hline $4 b_{1}$ & 50 & 25 & 50 & 25 & 25 & 50 \\
\hline $4 b_{2}$ & 25 & 25 & 50 & 25 & 50 & 25 \\
\hline $4 b_{3}$ & 25 & 25 & 50 & 25 & 25 & 50 \\
\hline $4 b_{4}$ & 50 & 25 & 50 & 25 & 50 & 25 \\
\hline $4 b_{5}$ & 25 & 25 & 25 & 25 & 25 & 25 \\
\hline $4 b_{6}$ & 25 & 25 & 25 & 25 & 25 & 25 \\
\hline Ciprofloxacin & 0.50 & 0.50 & 0.50 & 1.00 & -- & -- \\
\hline Clotrimazole & -- & -- & -- & -- & 1.00 & 3.00 \\
\hline DMF & -- & -- & -- & -- & -- & -- \\
\hline
\end{tabular}

aAverage of three readings; S. a: Staphylococcus aureus; S. f: Staphylococcus fecalis; E. c: Escherichia coli; S. $t$ : Salmonella typhi; C. a: Candida albicans; A. $n$ : Aspergillus niger.

form of a suspension ( $1 \%$ carboxymethyl cellulose as vehicle) by oral route. The study of analgesic activity was done by tail immersion method, ${ }^{14}$ using wistar albino mice. The percent analgesic activity was calculated and listed in Table 3 with a standard drug indomethacin for comparison. Compounds $\mathbf{4} \mathbf{a}_{2}, \mathbf{a}_{\mathbf{4}}$, and $\mathbf{4} \mathbf{b}_{\mathbf{2}}, \mathbf{b}_{\mathbf{4}}$ having diethylaminomethyl and dihexylaminomethyl groups, respectively at $1^{\prime}$ position of 2,3-indolinedione nucleus showed significant percent analgesic activity ranging from 50 to 54.96 after $1 \mathrm{~h}$ of administration. Moreover, diethylaminomethyl group was found to be optimum for analgesic activity in comparison to other groups. It was noted that bromination at 6 position of 3-methylquinoxaline-4(3H)-one ring decreases the analgesic activity. Remaining compounds showed less analgesic activity in comparison to standard drug indomethacin.

In the present investigation the anti-inflammatory activity of test compounds $\mathbf{4} \mathbf{a}_{\mathbf{1 - 6}}$ and $\mathbf{4 b}_{\mathbf{1 - 6}}$ in acute conditions was evaluated using the carrageenan induced rat paw edema method of Winter et al. ${ }^{15}$ The carrageenan induced inflammation model is a cyclooxygenases(COX) dependent reaction and is used to determine COX inhibition. For anti-inflammatory evaluation, adult 
Table 3. Analgesic activities of orally administered compounds $\mathbf{4 a}_{\mathbf{1 - 6}}$ and $\mathbf{4} \mathbf{b}_{\mathbf{1 - 6}}$ by tail immersion method

\begin{tabular}{|c|c|c|c|c|c|}
\hline \multirow{2}{*}{ Compd. No. } & \multirow{2}{*}{ Dose $\mathrm{mg} \mathrm{kg}^{-1}$} & \multicolumn{4}{|c|}{ Percentage analgesic activity } \\
\hline & & $30 \mathrm{~min}$ & $1 \mathrm{~h}$ & $2 \mathrm{~h}$ & $3 \mathrm{~h}$ \\
\hline $4 a_{1}$ & 100 & $27.33 \pm 0.017^{\mathrm{a}}$ & $37.20 \pm 0.125$ & $22.01 \pm 0.008$ & $16.72 \pm 0.014^{\mathrm{a}}$ \\
\hline $4 a_{2}$ & 100 & $50.45 \pm 0.017^{\mathrm{a}}$ & $53.58 \pm 0.011^{\mathrm{c}}$ & $34.52 \pm 0.005^{\mathrm{a}}$ & $23.46 \pm 0.011$ \\
\hline $4 a_{3}$ & 100 & $44.22 \pm 0.010^{c}$ & $47.06 \pm 0.007^{c}$ & $26.46 \pm 0.011^{\mathrm{b}}$ & $18.30 \pm 0.011^{\mathrm{c}}$ \\
\hline $4 a_{4}$ & 100 & $47.32 \pm 0.012^{\mathrm{a}}$ & $52.41 \pm 0.009^{\mathrm{a}}$ & $29.05 \pm 0.011^{\mathrm{b}}$ & $17.87 \pm 0.006^{\mathrm{c}}$ \\
\hline $4 a_{5}$ & 100 & $46.01 \pm 0.012^{\mathrm{a}}$ & $49.87 \pm 0.013^{\mathrm{a}}$ & $32.11 \pm 0.004^{\mathrm{a}}$ & $21.52 \pm 0.008^{a}$ \\
\hline $4 a_{6}$ & 100 & $32.12 \pm 0.016^{\mathrm{a}}$ & $36.01 \pm 0.005^{\mathrm{a}}$ & $25.40 \pm 0.010^{\mathrm{b}}$ & $14.72 \pm 0.008^{\mathrm{a}}$ \\
\hline $4 b_{1}$ & 100 & $29.44 \pm 0.017^{\mathrm{a}}$ & $35.55 \pm 0.017^{\mathrm{a}}$ & $21.45 \pm 0.015^{\mathrm{a}}$ & $19.21 \pm 0.032^{\mathrm{a}}$ \\
\hline $4 b_{2}$ & 100 & $53.74 \pm 0.006^{\mathrm{a}}$ & $54.96 \pm 0.012^{\mathrm{b}}$ & $28.10 \pm 0.008^{\mathrm{b}}$ & $21.04 \pm 0.008^{b}$ \\
\hline $4 b_{3}$ & 100 & $43.15 \pm 0.008^{\mathrm{a}}$ & $49.71 \pm 0.015^{b}$ & $30.12 \pm 0.011^{\mathrm{b}}$ & $21.58 \pm 0.012^{\mathrm{a}}$ \\
\hline $4 b_{4}$ & 100 & $47.12 \pm 0.011$ & $50.05 \pm 0.008^{b}$ & $25.82 \pm 0.013^{b}$ & $24.33 \pm 0.017^{b}$ \\
\hline $4 b_{5}$ & 100 & $46.87 \pm 0.012^{b}$ & $49.38 \pm 0.014^{\mathrm{b}}$ & $30.27 \pm 0.007^{c}$ & $20.73 \pm 0.011^{\mathrm{c}}$ \\
\hline $4 b_{6}$ & 100 & $39.55 \pm 0.031^{b}$ & $47.34 \pm 0.010^{\mathrm{b}}$ & $26.32 \pm 0.006^{a}$ & $20.12 \pm 0.012^{\mathrm{a}}$ \\
\hline Indomethacin & 10 & $58.57 \pm 0.020^{c}$ & $78.53 \pm 0.061^{\mathrm{c}}$ & $72.02 \pm 0.073^{\mathrm{c}}$ & $73.27 \pm 0.004^{c}$ \\
\hline
\end{tabular}

Results are expressed in mean \pm SEM $(\mathrm{n}=6)$. Significance levels ${ }^{\mathrm{a}} \mathrm{P}<0.05$, ${ }^{\mathrm{b}} \mathrm{P}<0.01$, and ${ }^{\mathrm{c}} \mathrm{P}<0.001$ as compared with respective control.

albino rats of either sex were divided in groups of 6 . The percent anti-inflammatory activity with indomethacin as a standard drug is presented in Table 4. Compounds $\mathbf{4} \mathbf{a}_{2-} \mathbf{a}_{\mathbf{4}}$ and $\mathbf{4} \mathbf{a}_{6}$ exhibited significant percent antiinflammatory activity ranging from 43.14 to 46.85 after $1 \mathrm{~h}$ of administration while remaining compounds showed lower activity in comparison to indomethacin. It was observed that compounds $\mathbf{4} \mathbf{a}_{\mathbf{3}}$ and $\mathbf{4} \mathbf{a}_{\mathbf{4}}$ having bulky diphenylaminomethyl and dihexylaminomethyl groups, respectively at $1^{\prime}$ position of 2,3-indolinedione nucleus showed maximum activity. It is evident from Table 4 that bromination at 6 position of 3-methylquinoxalin-4(3H)one nucleus decreases the anti-inflammatory activity.

Some of the compounds tested produced significant analgesic and anti-inflammatory effects. These compounds may exhibit both central and peripheral actions because analgesia and inflammation are central and peripheral processes, respectively. The compounds show suppressant activity on acute inflammation model of carrageenan induced inflammation in rats paw and produced significant analgesic activity. From these observations it can be indeed inferred that the endogenous chemical substances liberated during pain and inflammation like histamine, serotonin and arachidonic cascade metabolites are inhibited to produce analgesic and anti-inflammatory activities.

\section{Antihelmintic activity}

The antihelmintic activity of the synthesized compounds $\mathbf{4} \mathbf{a}_{\mathbf{1 - 6}}$ and $\mathbf{4} \mathbf{b}_{1-6}$ were evaluated on adult Indian earthworm, Pheretima posthuma due to its anatomical and physiological resemblance with the intestinal roundworm

Table 4. Anti-inflammatory activities of orally administered compounds $\mathbf{4} \mathbf{a}_{\mathbf{1 - 6}}$ and $\mathbf{4} \mathbf{b}_{\mathbf{1 - 6}}$ by carrageenan induced rat paw edema method

\begin{tabular}{|c|c|c|c|c|c|}
\hline \multirow{2}{*}{ Compd. No. } & \multirow{2}{*}{ Dose $\mathrm{mg} \mathrm{kg}^{-1}$} & \multicolumn{4}{|c|}{ Percentage anti-inflammatory } \\
\hline & & $30 \mathrm{~min}$ & $1 \mathrm{~h}$ & $2 \mathrm{~h}$ & $3 \mathrm{~h}$ \\
\hline$\overline{4 a_{1}}$ & 100 & $28.31 \pm 0.007^{b}$ & $33.76 \pm 0.011^{\mathrm{c}}$ & $31.11 \pm 0.007^{\mathrm{b}}$ & $29.32 \pm 0.017^{\mathrm{a}}$ \\
\hline $4 a_{2}$ & 100 & $38.03 \pm 0.017^{\mathrm{a}}$ & $41.68 \pm 0.021^{\mathrm{c}}$ & $39.41 \pm 0.008^{\mathrm{a}}$ & $36.63 \pm 0.011^{\mathrm{a}}$ \\
\hline $4 a_{3}$ & 100 & $44.01 \pm 0.015^{\mathrm{b}}$ & $46.85 \pm 0.008^{b}$ & $39.56 \pm 0.011^{\mathrm{b}}$ & $31.12 \pm 0.006^{\mathrm{b}}$ \\
\hline $4 a_{4}$ & 100 & $43.38 \pm 0.011^{\mathrm{a}}$ & $46.69 \pm 0.008^{b}$ & $38.27 \pm 0.004^{b}$ & $32.31 \pm 0.008^{\mathrm{b}}$ \\
\hline $4 a_{5}$ & 100 & $36.71 \pm 0.006$ & $43.28 \pm 0.003^{\mathrm{a}}$ & $33.74 \pm 0.007$ & $29.80 \pm 0.007^{\mathrm{a}}$ \\
\hline $4 a_{6}$ & 100 & $39.25 \pm 0.011^{\mathrm{a}}$ & $43.14 \pm 0.008^{c}$ & $31.23 \pm 0.007^{\mathrm{b}}$ & $23.43 \pm 0.006^{\mathrm{a}}$ \\
\hline $4 b_{1}$ & 100 & $31.86 \pm 0.017$ & $35.76 \pm 0.008^{b}$ & $27.84 \pm 0.012^{\mathrm{a}}$ & $24.58 \pm 0.011^{\mathrm{a}}$ \\
\hline $4 b_{2}$ & 100 & $19.76 \pm 0.013$ & $26.82 \pm 0.007^{b}$ & $23.09 \pm 0.006^{\mathrm{b}}$ & $20.41 \pm 0.011^{\mathrm{b}}$ \\
\hline $4 b_{3}$ & 100 & $29.44 \pm 0.011^{\mathrm{a}}$ & $33.17 \pm 0.011^{\mathrm{b}}$ & $32.38 \pm 0.023^{b}$ & $26.94 \pm 0.003^{b}$ \\
\hline $4 b_{4}$ & 100 & $29.22 \pm 0.008$ & $35.63 \pm 0.004^{b}$ & $32.29 \pm 0.011^{\mathrm{a}}$ & $28.53 \pm 0.010^{\mathrm{a}}$ \\
\hline $4 b_{5}$ & 100 & $23.05 \pm 0.011^{\mathrm{b}}$ & $30.71 \pm 0.011^{b}$ & $26.43 \pm 0.011^{\mathrm{a}}$ & $22.57 \pm 0.011^{\mathrm{a}}$ \\
\hline $4 b_{6}$ & 100 & $41.57 \pm 0.003^{\mathrm{a}}$ & $45.42 \pm 0.022^{c}$ & $28.33 \pm 0.011^{\mathrm{a}}$ & $24.11 \pm 0.013^{\mathrm{a}}$ \\
\hline Indomethacin & 10 & $52.94 \pm 0.023^{\mathrm{c}}$ & $55.69 \pm 0.023^{c}$ & $50.00 \pm 0.005^{c}$ & $44.68 \pm 0.009^{c}$ \\
\hline
\end{tabular}

Results are expressed in mean \pm SEM $(n=6)$ Significance levels ${ }^{a} \mathrm{P}<0.05,{ }^{\mathrm{b}} \mathrm{P}<0.01$ and ${ }^{\mathrm{c}} \mathrm{P}<0.001$ as compared with the respective control. 
parasites of human beings. ${ }^{16-18}$ The method of Dash et $a l .{ }^{19}$ was followed and the results are presented in Table 5. Piperazine citrate, which is used in the treatment of roundworm and threadworm (cestoda) infections was selected as a reference standard. The antihelmintic activity data revealed significant activity of all the test compounds against the tested Pheretima posthuma. In the present study compounds $\mathbf{4} \mathbf{a}_{\mathbf{3 - 6}}$ and $\mathbf{4} \mathbf{b}_{5,6}$ were found to be more potent than piperazine citrate. It is evident from results that compounds containing dihexylaminomethyl, piperazinylmethyl and morpholinomethyl groups at $1^{\prime}$ position of 2,3-indolinedione ring are more active. It is also noted that bromination at 6 position of 2-methylquinazolin$4(3 H)$-one derivatives slightly increases the antihelmintic activity. The principal aim of the present study was to modify and optimize the structural features of 2,3 , 6-trisubstituted quinazolin-4(3H)-ones.

Table 5. Antihelmintic activities of compounds $\mathbf{4} \mathbf{a}_{\mathbf{1 - 6}}$ and $\mathbf{4} \mathbf{b}_{\mathbf{1 - 6}}$

\begin{tabular}{lcccc}
\hline Group & Compound no. & $\begin{array}{c}\text { Conc./ } \\
\left(\mathrm{mg} \mathrm{mL}^{-1}\right)\end{array}$ & $\begin{array}{c}\text { Time taken for } \\
\text { paralysis } /\left(\mathrm{min}^{\mathrm{a}}\right)\end{array}$ & $\begin{array}{c}\text { Time taken for } \\
\text { death } /\left(\mathrm{min}^{\mathrm{a}}\right)\end{array}$ \\
\hline 1 & $\mathbf{4 a}_{\mathbf{1}}$ & 15 & 30 & 60 \\
2 & $\mathbf{4 a}_{\mathbf{2}}$ & 15 & 27 & 47 \\
3 & $\mathbf{4 a}_{\mathbf{3}}$ & 15 & 26 & 58 \\
4 & $\mathbf{4 a}_{\mathbf{4}}$ & 15 & 20 & 49 \\
5 & $\mathbf{4 a}_{\mathbf{5}}$ & 15 & 22 & 47 \\
6 & $\mathbf{4 a}_{\mathbf{6}}$ & 15 & 23 & 48 \\
7 & $\mathbf{4 b}_{\mathbf{1}}$ & 15 & 32 & 61 \\
8 & $\mathbf{4 b}_{\mathbf{2}}$ & 15 & 27 & 54 \\
9 & $\mathbf{4 b}_{\mathbf{3}}$ & 15 & 25 & 51 \\
10 & $\mathbf{4 b}_{\mathbf{4}}$ & 15 & 33 & 61 \\
11 & $\mathbf{4 b}_{\mathbf{5}}$ & 15 & 15 & 41 \\
12 & $\mathbf{4 b}_{\mathbf{6}}$ & 15 & 17 & 37 \\
13 & Control $_{4}$ & - & - & - \\
14 & Piperazine- & 15 & 28 & 62 \\
\hline
\end{tabular}

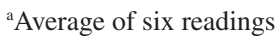

\section{Conclusions}

Generally, it can be concluded that $3-\left\{\left(1^{\prime}\right.\right.$-aryl/ alkylaminomethyl-2'-oxo- $1^{\prime}, 2^{\prime}$-dihydroindole-3'-ylidene) amino -2-methyl-6- quinazolin-4-(3H)-ones $\mathbf{4} \mathbf{a}_{\mathbf{1 - 6}}$ and $\mathbf{4} \mathbf{b}_{\mathbf{1 - 6}}$ represent a new distinct classes of analgesic and anti-inflammatory agents with antimicrobial activity. The dibenzylaminomethyl $\mathbf{4} \mathbf{a}_{\mathbf{3}}$, dihexylaminomethyl $\mathbf{4} \mathbf{a}_{\mathbf{4}}$ and morpholinomethyl $\mathbf{4} \mathbf{b}_{\mathbf{6}}$ analogs appeared to be more active analgesic and anti-inflammatory agents in comparison to other synthesized Schiff bases. Compound $\mathbf{4} \mathbf{b}_{\mathbf{6}}$, which contain morpholinomethyl moiety at $1^{\prime}$ position, was found to be the most active compound as analgesic, anti-inflammatory and antimicrobial agent. Antihelmintic study results revealed that the presence of piperazinylmethyl and morpholinomethyl groups at $1^{\prime}$ position of 2,3-indolinedione nucleus and a bromine atom at 6 position of 2-quinazolin-4 $(3 \mathrm{H})$-one ring are optimum for the antihelmintic activity $\left(\mathbf{4} \mathbf{b}_{5}\right.$ and $\left.\mathbf{4} \mathbf{b}_{\mathbf{6}}\right)$.

\section{Experimental}

Melting points were determined on a Tempstar apparatus and are uncorrected. Infrared spectra were recorded on a Jasco (410) FT-infrared spectrophotometer, measured as $\mathrm{KBr}$ disks. ${ }^{1} \mathrm{H}$ NMR were recorded on a Bruker DPX-300 MHz spectrometer in deuteriochloroform with trimethylsilane as internal standard (chemical shift in $\delta$ ppm). The mass spectral data were obtained with a Perkin-Elmer Hitachi RMU-6L MS-30 spectrometer at $70 \mathrm{eV}$ and a $90{ }^{\circ} \mathrm{C}$ inlet temperature. Purity of all the compounds was checked on silica gel plates and spots were located in iodine vapours. Elemental analysis was performed on EURO EA (Italy) analyzer and the results were within $\pm 0.3 \%$ of calculated values.

\section{General method for the preparation of Schiff bases $(3 \boldsymbol{a}, \boldsymbol{b})$}

To a solution of 2,3-indolinedione $(0.005 \mathrm{~mol})$ in ethanol $(50 \mathrm{~mL})$ was added the appropriate 3-amino-2methylquinazolin-4(3H)-one $\mathbf{2 a , b}(0.005 \mathrm{~mol})$ and a few drops of acetic acid. The reaction mixture was refluxed for $17 \mathrm{~h}$ on a water bath and then allowed to cool. The solid thus separated was filtered, washed with aqueous ethanol and recrystallized from appropriate solvent. The following Schiff bases were prepared in this manner:

3-\{(2'-Oxo-1',2'-dihydroindole-3'-ylidene $)$ amino $\}-2-$ methylquinazolin-4-(3H)-one (3a)

Recryst. solvent: Benzene, Yield (1.110 g, 73\%), mp $112{ }^{\circ} \mathrm{C}$. Elemental Analysis Calculated for $\mathrm{C}_{17} \mathrm{H}_{12} \mathrm{~N}_{4} \mathrm{O}_{2}$ (304.30): 61.10\% C; $3.97 \%$ H; $18.41 \%$ N. Found: $60.97 \%$ C; $4.22 \% \mathrm{H} ; 18.03 \% \mathrm{~N} .{ }^{1} \mathrm{H} \mathrm{NMR} \delta 2.50\left(\mathrm{~s}, 3 \mathrm{H}, \mathrm{CH}_{3}\right), 6.21-8.57$ (m, 8H, ArH), 10.63 (s, 1H, NH). MS m/z $304\left(\mathrm{M}^{+}\right)$. FT-IR $(\mathrm{KBr}), v_{\max } / \mathrm{cm}^{-1}: 3472(\mathrm{~N}-\mathrm{H}), 3076(\mathrm{Ar}-\mathrm{H}), 1727(\mathrm{C}=\mathrm{O})$, $1670(\mathrm{C}=\mathrm{O}), 1616(\mathrm{C}=\mathrm{N}), 1588(\mathrm{C}=\mathrm{N}), 1300(\mathrm{C}-\mathrm{N})$.

\footnotetext{
6-Bromo-3-\{(2'-oxo-1', 2'-dihydroindole-3'-ylidene) amino\}-2-methylquinazolin-4-(3H)-one (3b)

Recryst. solvent: Benzene, Yield (1.379 g, 72\%), mp $121{ }^{\circ} \mathrm{C}$. Elemental Analysis Calculated for $\mathrm{C}_{17} \mathrm{H}_{11} \mathrm{~N}_{4} \mathrm{O}_{2} \mathrm{Br}$ (383.19): $53.28 \%$ C; $2.89 \%$ H; $14.62 \%$ N. Found: $53.47 \%$ $\mathrm{C} ; 3.09 \% \mathrm{H} ; 14.23 \% \mathrm{~N} .{ }^{1} \mathrm{H}$ NMR $\delta 2.48\left(\mathrm{~s}, 3 \mathrm{H}, \mathrm{CH}_{3}\right)$, 6.38-8.49 (m, 7H, ArH), 10.53 (s, 1H, NH). MS (m/z) 383 $\left(\mathrm{M}^{+}\right)$. FT-IR $(\mathrm{KBr}), v_{\max } / \mathrm{cm}^{-1}: 3472(\mathrm{~N}-\mathrm{H}), 3076(\mathrm{Ar}-\mathrm{H})$, $1727(\mathrm{C}=\mathrm{O}), 1670(\mathrm{C}=\mathrm{O}), 1616(\mathrm{C}=\mathrm{N}), 1588(\mathrm{C}=\mathrm{N})$, $1300(\mathrm{C}-\mathrm{N})$.
} 
General method for the preparation of Mannich bases $4 a_{1-6}$ and $4 b_{1-6}$

To a mixture of 3a,b $(0.005 \mathrm{~mol})$ and $37 \%$ formalin $(1$ $\mathrm{mL})$ in ethanol $(20 \mathrm{~mL})$ was added drop wise appropriate secondary amines $(0.005 \mathrm{~mol})$ with stirring over $15 \mathrm{~min}$. The stirring was continued for $1 \mathrm{~h}$ at room temperature and the reaction mixture then warmed for $15 \mathrm{~min}$ on a water bath. The mixture was poured into ice-cold water and stored in a refrigerator. The solid thus separated was filtered, washed with water, dried and recrystallized from appropriate solvent. The following title compounds were prepared as just described:

3-\{(1'-Dimethylaminomethyl-2'-oxo- $1^{\prime}, 2^{\prime}$-dihydroindole- $3^{\prime}$ ylidene)amino -2-methyl-6- quinazolin-4-(3H)-one (4a $)$

Recryst. solvent: Ethanol, Yield (1.138 g, 63\%), mp $150{ }^{\circ} \mathrm{C}$. Elemental Analysis Calculated for $\mathrm{C}_{20} \mathrm{H}_{19} \mathrm{~N}_{5} \mathrm{O}_{2}$ (361.39): $66.47 \%$ C; $5.30 \%$ H; $19.38 \%$ N. Found: $66.32 \%$ $\mathrm{C} ; 5.41 \% \mathrm{H} ; 19.31 \% \mathrm{~N} .{ }^{1} \mathrm{H}$ NMR $\delta 1.12,\left(\mathrm{~s}, 3 \mathrm{H}, \mathrm{CH}_{3}\right)$, 2.5 (s, 6H, N ( $\left.\left.\mathrm{CH}_{3}\right)_{2}\right), 4.43$ (s, 2H, $\left.\mathrm{CH}_{2}\right), 6.5-8.98$ (m, 8H, ArH ). MS $(m / z) 361.39\left(\mathrm{M}^{+}\right)$. FT-IR $(\mathrm{KBr}), v_{\max } / \mathrm{cm}^{-1}$ : $3026(\mathrm{Ar}-\mathrm{H}), 2816\left(\mathrm{CH}_{2}\right), 1733(\mathrm{C}=\mathrm{O}), 1662(\mathrm{C}=\mathrm{O}), 1570$ $(\mathrm{C}=\mathrm{N}), 1317(\mathrm{C}-\mathrm{N})$.

3-\{(1'-Diethylaminomethyl-2'-oxo- $1^{\prime}, 2^{\prime}$-dihydroindole- $3^{\prime}$ ylidene)amino\}-2-methyl-6-quinazolin-4-(3H)-one (4a)

Recryst. solvent: Ethanol, Yield (1.479 g, 76\%), mp $142{ }^{\circ} \mathrm{C}$. Elemental Analysis Calculated for $\mathrm{C}_{22} \mathrm{H}_{23} \mathrm{~N}_{5} \mathrm{O}_{2}$ (389.45): $67.85 \%$ C; $5.95 \%$ H; $17.98 \%$ N. Found: $67.88 \%$ $\mathrm{C} ; 5.87 \% \mathrm{H} ; 18.10 \% \mathrm{~N} .{ }^{1} \mathrm{H} \mathrm{NMR} \delta 1.09$ (s, 3H, $\left.\mathrm{CH}_{3}\right)$, $\left.2.55\left(\mathrm{q}, 10 \mathrm{H}, \mathrm{N}\left(\mathrm{C}_{2} \mathrm{H}_{5}\right)_{2}\right), 2.88\left(\mathrm{~s}, 3 \mathrm{H}, \mathrm{CH}_{3}\right),\right), 4.32(\mathrm{~s}, 2 \mathrm{H}$, $\left.\mathrm{CH}_{2}\right)$, 6.57-8.22 (m, 8H, ArH). MS (m/z) $389.40\left(\mathrm{M}^{+}\right)$. FT-IR (KBr), $v_{\max } / \mathrm{cm}^{-1}: 3030(\mathrm{Ar}-\mathrm{H}), 2855\left(\mathrm{CH}_{2}\right), 1717$ $(\mathrm{C}=\mathrm{O}), 1661(\mathrm{C}=\mathrm{O}), 1609(\mathrm{C}=\mathrm{N}), 1350(\mathrm{C}-\mathrm{N})$.

3-\{(1'-Diphenylaminomethyl-2'-oxo- $1^{\prime}, 2^{\prime}$-dihydroindole3'-ylidene)amino -2-methyl-6-quinazolin-4-(3H)-one $\left(4 a_{3}\right)$

Recryst. solvent: Ethanol, Yield (10.769 g, 52\%), mp $140{ }^{\circ} \mathrm{C}$. Elemental Analysis Calculated for $\mathrm{C}_{30} \mathrm{H}_{23} \mathrm{~N}_{5} \mathrm{O}_{2}$ (485.53): $74.21 \%$ C; $4.77 \%$ H; $14.42 \%$ N. Found: $74.13 \%$ $\mathrm{C} ; 4.74 \% \mathrm{H} ; 14.45 \%$ N. ${ }^{1} \mathrm{H}$ NMR $\delta 1.09$ (s, 3H, $\mathrm{CH}_{3}$ ), 5.10 $\left(\mathrm{s}, 2 \mathrm{H}, \mathrm{CH}_{2}\right), 6.36-8.20(\mathrm{~m}, 18 \mathrm{H}, \mathrm{ArH})$. Mass spectrum $(\mathrm{m} / \mathrm{z}) 485.50\left(\mathrm{M}^{+}\right)$. FT-IR $(\mathrm{KBr}), v_{\max } / \mathrm{cm}^{-1}: 3032(\mathrm{Ar}-\mathrm{H})$, $2841\left(\mathrm{CH}_{2}\right), 1742(\mathrm{C}=\mathrm{O}), 1689(\mathrm{C}=\mathrm{O}), 1636(\mathrm{C}=\mathrm{N}), 1334$ (C-N).

3-\{(1'-Dihexylaminomethyl-2'-oxo- $1^{\prime}, 2^{\prime}$-dihydroindole- $3^{\prime}$ ylidene)amino \}-2-methyl-6-quinazolin-4-(3H)-one (4a $)$

Recryst. solvent: Ethanol, Yield (1.517 g, 61\%), mp
$152{ }^{\circ} \mathrm{C}$. Elemental Analysis Calculated for $\mathrm{C}_{30} \mathrm{H}_{35} \mathrm{~N}_{5} \mathrm{O}_{2}$ (497.63): $72.41 \%$ C; $7.09 \%$ H; $14.07 \%$ N. Found: $72.49 \%$ $\mathrm{C} ; 6.97 \% \mathrm{H} ; 14.21 \%$ N. ${ }^{1} \mathrm{H}$ NMR $\delta 1.59$ (s, 3H, $\left.\mathrm{CH}_{3}\right), 1.73$ (t, 2H, $\mathrm{CH}_{2}$ ), 3.91 (s, 22H, $\mathrm{CH}_{2}$ ), 7.00-8.66 (m, 8H, ArH). $\operatorname{MS}(\mathrm{m} / \mathrm{z}) 497.63\left(\mathrm{M}^{+}\right)$. FT-IR (KBr), $v_{\max } / \mathrm{cm}^{-1}: 3076(\mathrm{Ar}-\mathrm{H})$, $2881\left(\mathrm{CH}_{2}\right), 1733(\mathrm{C}=\mathrm{O}), 1670(\mathrm{C}=\mathrm{O}), 1607(\mathrm{C}=\mathrm{N}), 1307$ (C-N).

3-\{(1'-Piperazinylaminomethyl-2'-oxo- $1^{\prime}, 2^{\prime}$-dihydroindole3'-ylidene)amino\}-2-methyl-6-quinazolin-4-(3H)-one $\left(4 a_{5}\right)$

Recryst. solvent: Ethanol, Yield (1.817 g, 59\%), mp 154 ${ }^{\circ} \mathrm{C}$. Elemental Analysis Calculated for $\mathrm{C}_{22} \mathrm{H}_{22} \mathrm{~N}_{6} \mathrm{O}_{2}$ (402.44): $65.66 \% \mathrm{C} ; 5.51 \% \mathrm{H} ; 20.88 \%$ N. Found $65.42 \% \mathrm{C} ; 5.80 \%$ $\mathrm{H} ; 20.67 \%$ N. ${ }^{1} \mathrm{H}$ NMR $\delta 1.21\left(\mathrm{~s}, 3 \mathrm{H}, \mathrm{CH}_{3}\right), 2.63$ (s, 2H, $\left.\mathrm{CH}_{2}\right), 4.00\left(\mathrm{t}, 4 \mathrm{H}, \mathrm{CH}_{2}\right), 4.49\left(\mathrm{~m}, 4 \mathrm{H}, \mathrm{CH}_{2}\right), 6.70-8.20$ (m, $8 \mathrm{H}, \mathrm{ArH}), 8.37(\mathrm{~s}, 1 \mathrm{H}, \mathrm{NH})$. MS $(\mathrm{m} / \mathrm{z}) 402.39\left(\mathrm{M}^{+}\right)$. FT-IR $(\mathrm{KBr}), v_{\max } / \mathrm{cm}^{-1}: 3361(\mathrm{NH}), 3013(\mathrm{Ar}-\mathrm{H}), 2888\left(\mathrm{CH}_{2}\right)$, $1687(\mathrm{C}=\mathrm{O}), 1652(\mathrm{C}=\mathrm{O}), 1602(\mathrm{C}=\mathrm{N}), 1325(\mathrm{C}-\mathrm{N})$.

3-\{( $1^{\prime}$-Morpholinoaminomethyl-2'-oxo- $1^{\prime}, 2^{\prime}$-dihydroindole3'-ylidene)amino\}-2-methyl-6-quinazolin-4-(3H)-one $\left(4 a_{6}\right)$

Recryst. solvent: Ethanol, Yield (1.35 g, 67\%), mp $141{ }^{\circ} \mathrm{C}$. Elemental Analysis Calculated for $\mathrm{C}_{22} \mathrm{H}_{21} \mathrm{~N}_{5} \mathrm{O}_{3} \mathrm{Br}$ (403.42): $65.50 \%$ C; $5.25 \%$ H; $17.36 \%$ N. Found $65.75 \%$ $\mathrm{C} ; 5.55 \% \mathrm{H} ; 17.06 \% \mathrm{~N} .{ }^{1} \mathrm{H}$ NMR $\delta 1.06\left(\mathrm{~s}, 3 \mathrm{H}, \mathrm{CH}_{3}\right)$, $2.37\left(\mathrm{~s}, 2 \mathrm{H}, \mathrm{CH}_{2}\right), 3.40\left(\mathrm{t}, 4 \mathrm{H}, \mathrm{CH}_{2}\right), 5.75\left(\mathrm{t}, 4 \mathrm{H}, \mathrm{CH}_{2}\right)$, 6.66-8.36 (m, 8H, ArH ), 8.58(s,1H, NH). MS ( $/ 2 / z) 403.42$ $\left(\mathrm{M}^{+}\right)$. FT-IR (KBr), $v_{\max } / \mathrm{cm}^{-1}: 3066(\mathrm{Ar}-\mathrm{H}), 2818\left(\mathrm{CH}_{2}\right)$, 1726(C=O), $1675(\mathrm{C}=\mathrm{O}), 1604(\mathrm{C}=\mathrm{N}), 1314(\mathrm{C}-\mathrm{N}) 1128$ (COC), 632(C-Br).

6-Bromo-3-\{( $1^{\prime}$-dimethylaminomethyl-2'-oxo- $1^{\prime}, 2^{\prime}$ dihydroindole-3'-ylidene)amino\}-2-methyl-6-quinazolin4-(3H)-one $\left(\mathbf{4} \boldsymbol{b}_{1}\right)$

Recryst. solvent: Ethanol, Yield (1.089g, 69\%), mp $165{ }^{\circ} \mathrm{C}$. Elemental Analysis Calculated for $\mathrm{C}_{20} \mathrm{H}_{18} \mathrm{~N}_{5} \mathrm{O}_{2} \mathrm{Br}$ (440.29): $54.56 \%$ C; $4.12 \%$ H; $15.91 \%$ N. Found: $54.83 \%$ $\mathrm{C} ; 4.10 \% \mathrm{H} ; 15.78 \%$ N. ${ }^{1} \mathrm{H}$ NMR $\delta 1.06$ (s, 3H, $\left.\mathrm{CH}_{3}\right), 2.37$ $\left(\mathrm{t}, 3 \mathrm{H}, \mathrm{CH}_{3}\right), 3.40\left(\mathrm{t}, 3 \mathrm{H}, \mathrm{CH}_{3}\right), 5.57\left(\mathrm{~s}, 2 \mathrm{H}, \mathrm{CH}_{2}\right), 6.70-8.20$ $(\mathrm{m}, 7 \mathrm{H}, \mathrm{ArH}), 8.37(\mathrm{~s}, 1 \mathrm{H}, \mathrm{NH})$. MS $(\mathrm{m} / \mathrm{z}) 440.29\left(\mathrm{M}^{+}\right)$. FT-IR (KBr), $v_{\text {max }} / \mathrm{cm}^{-1}: 3066(\mathrm{Ar}-\mathrm{H}), 2818\left(\mathrm{CH}_{2}\right), 1726$ $(\mathrm{C}=\mathrm{O}), 1675(\mathrm{C}=\mathrm{O}), 1604(\mathrm{C}=\mathrm{N}), 1314(\mathrm{C}-\mathrm{N})$.

6-Bromo-3-\{( $1^{\prime}$-diethylaminomethyl-2' - oxo- $1^{\prime}, 2^{\prime}$ dihydroindole-3'-ylidene)amino -2-methyl-6-quinazolin4-(3H)-one $\left(4 b_{2}\right)$

Recryst. solvent: Ethanol , Yield (1.428 g, 61\%), mp $162{ }^{\circ} \mathrm{C}$. Elemental Analysis Calculated for $\mathrm{C}_{22} \mathrm{H}_{22} \mathrm{~N}_{5} \mathrm{O}$ Br (468.34): $56.42 \%$ C; $4.73 \%$ H; $14.95 \%$ N. Found: $56.47 \%$ 
$\mathrm{C} ; 4.83 \% \mathrm{H} ; 15.17 \%$ N. ${ }^{1} \mathrm{H}$ NMR $\delta 1.11\left(\mathrm{~s}, 3 \mathrm{H}, \mathrm{CH}_{3}\right)$, 2.50-2.87 (q, 10H, N $\left.\left(\mathrm{C}_{2} \mathrm{H}_{5}\right)_{2}\right), 4.29\left(\mathrm{~s}, 2 \mathrm{H}, \mathrm{CH}_{2}\right), 6.65-8.34$ $(\mathrm{m}, 7 \mathrm{H}, \mathrm{ArH}) . \mathrm{MS}(\mathrm{m} / \mathrm{z}) 468.34\left(\mathrm{M}^{+}\right)$. FT-IR $(\mathrm{KBr}$, $\left.v_{\max } / \mathrm{cm}^{-1}\right): 3032(\mathrm{Ar}-\mathrm{H}), 2853\left(\mathrm{CH}_{2}\right), 1717(\mathrm{C}=\mathrm{O}), 1663$ $(\mathrm{C}=\mathrm{O}), 1609(\mathrm{C}=\mathrm{N}), 1353(\mathrm{C}-\mathrm{N})$.

6-Bromo-3-\{(1'-diphenylaminomethyl-2'-oxo-1',2'dihydroindole-3'-ylidene)amino -2-methyl-6-quinazolin4-(3H)-one $\left(\mathbf{4} b_{3}\right)$

Recryst. solvent: Ethanol, Yield (1.777 g, 63\%), mp $144{ }^{\circ} \mathrm{C}$. Elemental Analysis Calculated for $\mathrm{C}_{30} \mathrm{H}_{22} \mathrm{~N}_{5} \mathrm{O}_{2} \mathrm{Br}$ (563.43): $63.84 \% \mathrm{C} ; 3.93 \% \mathrm{H} ; 12.41 \% \mathrm{~N}$. Found $64.10 \%$ $\mathrm{C} ; 4.22 \% \mathrm{H} ; 12.27 \%$ N. ${ }^{1} \mathrm{H}$ NMR $\delta 1.07$ (s, 3H, $\left.\mathrm{CH}_{3}\right)$, $4.98\left(\mathrm{~s}, 2 \mathrm{H}, \mathrm{CH}_{2}\right), 6.25-8.15(\mathrm{~m}, 17 \mathrm{H}, \mathrm{ArH}) . \mathrm{MS}(\mathrm{m} / \mathrm{z})$ $563.43\left(\mathrm{M}^{+}\right)$. FT-IR (KBr, $\left.v_{\max } / \mathrm{cm}^{-1}\right): 3032(\mathrm{Ar}-\mathrm{H})$, $2845\left(\mathrm{CH}_{2}\right), 1745(\mathrm{C}=\mathrm{O}), 1690(\mathrm{C}=\mathrm{O}), 1636(\mathrm{C}=\mathrm{N})$, 1334 (C-N).

6-Bromo-3-\{(1'-dihexylaminomethyl-2' -oxo- $1^{\prime}, 2^{\prime}$ dihydroindole-3'-ylidene)amino\}-2-methyl-6-quinazolin4- $(3 H)$-one $(\mathbf{4 b})$

Recryst. solvent: Ethanol, Yield (1.643 g, 57\%), mp $168{ }^{\circ} \mathrm{C}$. Elemental Analysis Calculated for $\mathrm{C}_{30} \mathrm{H}_{34} \mathrm{~N}_{5} \mathrm{O}_{2} \mathrm{Br}$ (576.52): $62.50 \% \mathrm{C} ; 5.94 \% \mathrm{H} ; 12.15 \%$ N. Found $62.41 \%$ $\mathrm{C} ; 5.73 \% \mathrm{H} ; 12.37 \% \mathrm{~N} .{ }^{1} \mathrm{H}$ NMR $\delta 1.06\left(\mathrm{~s}, 3 \mathrm{H}, \mathrm{CH}_{3}\right)$, $1.62\left(\mathrm{t}, 2 \mathrm{H}, \mathrm{CH}_{2}\right), 3.84\left(\mathrm{~s}, 22 \mathrm{H}, \mathrm{CH}_{2}\right), 7.22-8.56(\mathrm{~m}, 7 \mathrm{H}$, $\operatorname{ArH})$. MS $(\mathrm{m} / \mathrm{z}) 576.52\left(\mathrm{M}^{+}\right)$. FT-IR $\left(\mathrm{KBr}, v_{\max } / \mathrm{cm}^{-1}\right)$ : $3078(\mathrm{Ar}-\mathrm{H}), 2883\left(\mathrm{CH}_{2}\right), 1730(\mathrm{C}=\mathrm{O}), 1665(\mathrm{C}=\mathrm{O}), 1605$ $(\mathrm{C}=\mathrm{N}), 1307$ (C-N).

6-Bromo-3-\{(1'-piperazinylminomethyl-2'-oxo- $1^{\prime}, 2^{\prime}$ dihydroindole-3'-ylidene)amino\}-2-methyl-6-quinazolin4- $(3 H)$-one $\left(4 b_{5}\right)$

Recryst. solvent: Ethanol, Yield (1.492 g, 62\%), mp $164{ }^{\circ} \mathrm{C}$. Elemental Analysis Calculated for $\mathrm{C}_{22} \mathrm{H}_{21} \mathrm{~N}_{6} \mathrm{O}_{2} \mathrm{Br}$ (481.34): $54.90 \%$ C; $4.40 \%$ H; $17.46 \%$ N. Found: $54.83 \%$ $\mathrm{C} ; 4.62 \% \mathrm{H} ; 17.16 \%$ N. ${ }^{1} \mathrm{H}$ NMR $\delta 1.17$ (s, 3H, $\left.\mathrm{CH}_{3}\right), 2.55$ (s, 2H, $\left.\mathrm{CH}_{2}\right), 4.12\left(\mathrm{t}, 4 \mathrm{H}, \mathrm{CH}_{2}\right), 4.39\left(\mathrm{t}, 4 \mathrm{H}, \mathrm{CH}_{2}\right), 6.71-8.24$ $(\mathrm{m}, 7 \mathrm{H}, \mathrm{ArH}), 8.58(\mathrm{~s}, 1 \mathrm{H}, \mathrm{NH})$. MS (m/z) $481.34\left(\mathrm{M}^{+}\right)$. FTIR $\left(\mathrm{KBr}, v_{\max } / \mathrm{cm}^{-1}\right): 3357(\mathrm{NH}), 3017(\mathrm{Ar}-\mathrm{H}), 2876\left(\mathrm{CH}_{2}\right)$, $1687(\mathrm{C}=\mathrm{O}), 1643(\mathrm{C}=\mathrm{O}), 1600(\mathrm{C}=\mathrm{N}), 1317(\mathrm{C}-\mathrm{N})$.

6-Bromo-3- \{( $1^{\prime}$-morpholinoaminomethyl-2'-oxo- $1^{\prime}, 2^{\prime}$ dihydroindole-3'-ylidene)amino -2-methyl-6-quinazolin4-(3H)-one $\left(\mathbf{4} \boldsymbol{b}_{6}\right)$

Recryst. solvent: Ethanol, Yield (1.615 g, 67\%), mp $160{ }^{\circ} \mathrm{C}$. Elemental Analysis Calculated for $\mathrm{C}_{22} \mathrm{H}_{20} \mathrm{~N}_{5} \mathrm{O}_{3} \mathrm{Br}$ (482.32): $54.78 \% \mathrm{C} ; 4.18 \% \mathrm{H} ; 14.52 \% \mathrm{~N}$. Found $54.53 \%$ $\mathrm{C} ; 4.34 \% \mathrm{H} ; 14.29 \%$ N. ${ }^{1} \mathrm{H}$ NMR $\delta 1.06\left(\mathrm{~s}, 3 \mathrm{H}, \mathrm{CH}_{3}\right)$, $2.37\left(\mathrm{~s}, 2 \mathrm{H}, \mathrm{CH}_{2}\right), 3.40\left(\mathrm{t}, 4 \mathrm{H}, \mathrm{CH}_{2}\right), 5.75\left(\mathrm{t}, 4 \mathrm{H}, \mathrm{CH}_{2}\right)$, 6.66-8.36 (m, 7H, ArH), 8.58 (s, 1H, NH). MS (m/z) 482.32
$\left(\mathrm{M}^{+}\right)$. FT-IR $(\mathrm{KBr}), v_{\max } / \mathrm{cm}^{-1}: 3056(\mathrm{Ar}-\mathrm{H}), 2822\left(\mathrm{CH}_{2}\right)$, 1720 $(\mathrm{C}=\mathrm{O}), 1666(\mathrm{C}=\mathrm{O}), 1608(\mathrm{C}=\mathrm{N}), 1321(\mathrm{C}-\mathrm{N}), 1124$ (COC), 625 (C-Br).

\section{Conclusions}

Various 3- $\left\{\left(1^{\prime}\right.\right.$-aryl/alkylaminomethyl-2'-oxo- $1^{\prime}, 2^{\prime}$ dihydroindole-3'-ylidene)amino $\}$-2-methyl-6-quinazolin4-(3H)-ones $\mathbf{4} \mathbf{a}_{\mathbf{1 - 6}}$ and $\mathbf{4} \mathbf{b}_{\mathbf{1 - 6}}$ were prepared with the objective of developing better anti-inflammatory compounds with antimicrobial activities. Among these, 6-Bromo-3-\{(1'morpholinoaminomethyl-2'-oxo-1', $2^{\prime}$-dihydroindole-3'ylidene)amino -2-methyl-6-quinazolin-4-(3H)-one ( $\left.\mathbf{4} \mathbf{b}_{\mathbf{6}}\right)$ presented maximum activity. It also showed maximum antihelmintic activity. SAR studies and evaluation of more potent analogs are under way.

\section{Acknowledgments}

The authors are grateful to the authorities of University Department of Pharmaceutical Sciences, Utkal University, Bhubaneswar and Institute of Pharmacy and Technology, Salipur, Cuttack, Orissa, India for providing the necessary facility to carry out this research work. We are also thankful to Prof. A. C. Dash and Prof. C. S. Panda NISER, Bhubaneswar, India for valuable suggestions.

\section{References}

1. El-Shareif, A. M. S; Ammar, Y. A.; Zahran, M. A.; Ali, A. H.; El-Gaby, M. A.; Molecules 2001, 6, 267.

2. Sayyed, M. A.; Mokle, S. S.; Vibhute, Y. B.; Arkivoc 2006, 11, 221.

3. El-Barbary, A. A.; Abou El-Ezz, A. Z.; Sharaf, A. M.; Nielson, C.; Phosphorus, Sulfur, Silicon Relat. Elem. 2006, 18, 1895.

4. Srivastava, B.; Shukla, J. S.; Indian J. Chem. 1991, 30B, 332.

5. Pandeya, S. N.; Yogeeswari, P.; Ram, D. S.; Nath, G.; Boll. Chim. Farm. 1998, 137, 321.

6. Pandeya, S. N.; Sriram, D.; Nath, G.; De Clercq, E.; Pharm. Acta. Helv. 1999, 74, 11.

7. Pandeya, S. N.; Sriram, D.; Nath, G.; De Clercq, E.; Indian J. Pharm. Sci. 1999, 61, 358.

8. Sridhar, S. K.; Ramesh, A.; Biol. Pharm. Bull. 2001, 24, 1149.

9. Sriram, D.; Bal, T. R.; Perumal, Y.; J. Pharm. Pharmaceut. Sci. 2005, 8, 565 .

10. Wasfy, A. A. F; Indian J. Chem. 2003, 42B, 3102.

11. Yassin, F. A.; Eissa, A. M. F.; Wasfy, A. A. F.; Indian J. Chem. 1994, 33B, 1193.

12. Barry, A. L.; The Antimicrobial Susceptibility Test, Principle and Practices, $4^{\text {th }}$ ed., ELBS: London, 1999, 180. 
13. Dastidar, S. G.; Chaudhary, A.; Annadurai, S.; Roy, S.; Mookerjee, M.; Chakrabarty, A. N.; J. Chemother. 1995, 7, 201.

14. Di Stasi, L. C.; Costa, M.; Mendacolli, S. L. J.; Kirizawa, M.; Gomes, C.; Trolin, G.; J. Ethnopharmacol. 1988, 24, 205.

15. Winter, C. A.; Risly, E. A.; Nus, G. N.; Proc. Soc. Exp. Biol. 1962, 111, 544.

16. Thorn, G. W.; Adams, R. D.; Braunwald, E.; Isselbacher, K. J.; Petersdorf, R. G.; Harrison's principle of internal medicine, Mc Graw Hill Co.: New York, 1977, pp.1088.

17. Vigar, Z.; Atlas of Medicinal Parasitology, $2^{\text {nd }}$ ed., P. G. Publishing House: Singapore, 1984, pp. 216.
18. Szewczuk, V. D.; Mongelli, E. R.; Pamillio, A. B.; Mol. Med. Chem. 2003, 1,54

19. Dash, K. G.; Suresh, P.; Sahu, K. S.; Kar, D. M.; Ganapaty, S.; Panda. B. S.; J. Nat. Remedies 2002, 2, 182.

Received: September 22, 2007

Web Release Date: May 9, 2008 